\title{
Construcción del Sistema de Tutorado para Lúpulo y su Establecimiento en Florida ${ }^{1}$
}

\author{
Shinsuke Agehara, Aleyda Acosta-Rangel, Zhanao Deng, Jack Rechcigl, and Simon Bollin; \\ translated by Mariel Gallardo²
}

\section{Introducción}

El lúpulo (Humulus lupulus L.) es un ingrediente esencial en la elaboración de cerveza que le agrega amargura y sabor. Impulsado por el reciente movimiento de la cervecería artesanal, el volumen y la superficie de producción de lúpulo en EE. UU. aumentaron en un $62 \%$ y $78 \%$, respectivamente, del 2000 al 2019 (US Department of Agriculture-NASS 2019a). La superficie de producción en 2019 (55,800 acres o 22,581.46 hectáreas) fue la más alta registrada desde 1915 (US Department of Agriculture-NASS 2019a). La costa noroeste (es decir, Washington, Oregón e Idaho) suministra casi el 90\% del lúpulo producido en los Estados Unidos (International Hop Growers' Convention (IHGC) 2018; US Department of Agriculture-NASS 2019b). Actualmente, la producción de lúpulo se está expandiendo a estados no tradicionales en la producción de lúpulo, incluidos Colorado, California, Minnesota, Virginia y Carolina del Norte (Hop Growers of America 2019; Siegle 2020). En Florida, sin embargo, la producción comercial de lúpulo es casi inexistente

Según la Asociación de Cerveceros (Brewers Association 2018), la cantidad de cervecerías artesanales en Florida aumentó de 45 en 2011 a 285 en 2018, 95 de ellas se encuentran en el área de la bahía de Tampa. El impacto económico de la industria de cerveza artesanal en Florida se estima en más de \$3 mil millones. El primer campo de investigación de lúpulo se construyó en el UF/IFAS Gulf Coast Research and Education Center (GCREC) en 2016 (Figura 1A) para estudiar el lúpulo como un cultivo alternativo para Florida. En 2019, el campo de lúpulo se expandió de 0.8 a 2.2 acres o 0.32 a 0.89 hectáreas (Figura 1B). Un equipo interdisciplinario de más de 10 investigadores especializados en genética vegetal, fisiología vegetal, fitopatología, entomología, nematología y economía agrícola trabajan en la investigación del lúpulo en GCREC. Basándonos en nuestra experiencia con nuestro campo de lúpulo, preparamos este artículo con el objetivo de proporcionar pautas y consideraciones para construir el sistema de tutorado y establecer un campo de lúpulo en Florida.

\section{Características de Crecimiento y Requerimientos Climáticos}

Las plantas de lúpulo desarrollan tallos entrelazados. A diferencia de las enredaderas que trepan usando zarcillos u otros tejidos especializados, estos tallos se entrelazan con la ayuda de pelos rígidos (tricomas) distribuidos a lo largo de sus tallos. Los lúpulos crecen muy rápidamente, con un crecimiento máximo de hasta 12 " o $30.5 \mathrm{~cm}$ por día (Rebecca 2004). Debido a estas características de crecimiento,

1. Este documento, HS1354s, es uno de una serie del Departamento de Ciencias Hortícolas, UF/IFAS Extension de la Universidad de Florida. Fecha de publicación original: marzo 2021. Visite el sitio web de EDIS en https://edis.ifas.ufl.edu para obtener la versión actualizada de esta publicación.

2. Shinsuke Agehara, assistant professor, Horticultural Sciences Department; Aleyda Acosta-Rangel, postdoctoral associate; Zhanao Deng, professor, Environmental Horticulture Department; Jack Rechcigl, center director and professor, Department of Soil and Water Sciences; UF/IFAS Gulf Coast Research and Education Center; and Simon Bollin, agriculture industry development, Hillsborough County Economic Development Department; Translated by Mariel Gallardo, graduate research assistant, UF/IFAS Gulf Coast Research and Education Center, Balm, FL 33598.

The Institute of Food and Agricultural Sciences (IFAS) is an Equal Opportunity Institution authorized to provide research, educational information and other services

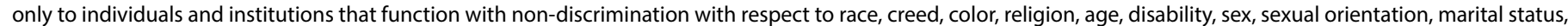

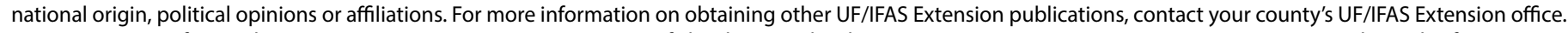
U.S. Department of Agriculture, UF/IFAS Extension Service, University of Florida, IFAS, Florida A \& M University Cooperative Extension Program, and Boards of County Commissioners Cooperating. Nick T. Place, dean for UF/IFAS Extension. 
las plantas de lúpulo cultivadas comercialmente son guiadas para crecer en un sistema de tutorado alto, generalmente de 12 a $18 \mathrm{ft}$ (3.7 a $5.5 \mathrm{~m}$ ) de altura (Dodds 2017). Los lúpulos comerciales se cosechan cortando los tallos en la base. Las coronas de las raíces permanecerán en el suelo, inactivas durante el invierno. Luego, a principios de la primavera se desarrollarán nuevos brotes.

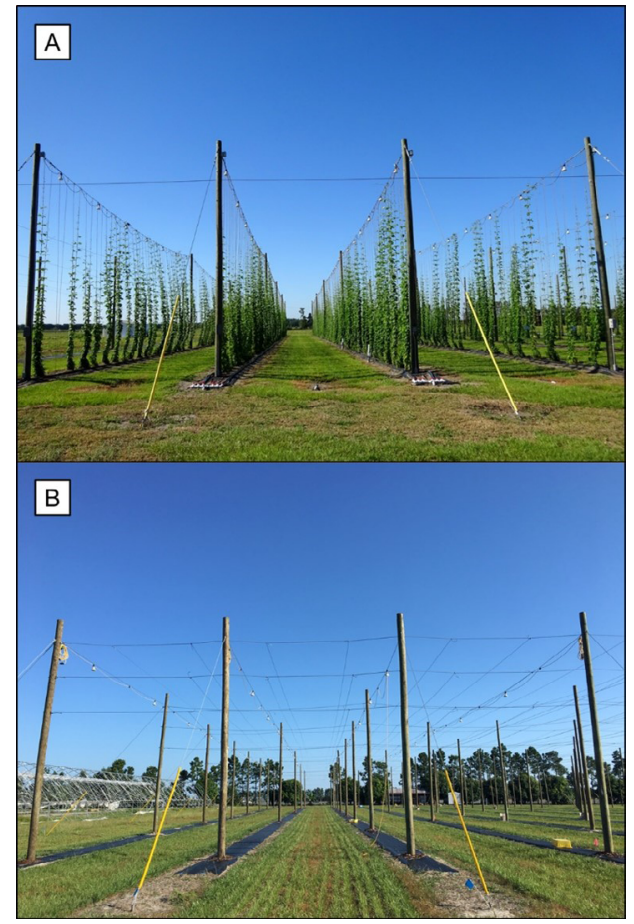

Figura 1. Campo de investigación de lúpulos de UF/IFAS GCREC: A) Tutorado recto, y B) Tutorado en "V".

Créditos: Shinsuke Agehara, UF/IFAS

La floración del lúpulo se activa al reducir las horas de luz por día (Thomas and Schwabe 1969). En el hemisferio norte, las plantas de lúpulo suelen florecer entre principios $\mathrm{y}$ mediados de julio, cuando el tiempo de luz se reduce a menos de 16 horas diarias (Iskra et al. 2019). Las plantas de lúpulo son más productivas cuando la cantidad de horas de luz diaria sostiene el buen crecimiento vegetativo antes de la inducción a floración. La duración de horas de luz está en función a la latitud, y el rango de latitud generalmente aceptado para la producción comercial de lúpulo es de $35^{\circ}$ a $55^{\circ}$ al norte o al sur línea ecuatorial (Dodds 2017). Las dos regiones de producción de lúpulo más grandes del mundo son Hallertau en Alemania y Yakima en los Estados Unidos, ubicadas a $48.6^{\circ} \mathrm{N}$ y $46.6^{\circ} \mathrm{N}$, respectivamente.

En Florida, la latitud es inferior a $31^{\circ} \mathrm{N}$ y la duración máxima de horas de luz no llega a las 15 horas diarias. Por lo tanto, las plantas de lúpulo que crecen bajo el periodo de luz natural de Florida tienden a florecer prematuramente, lo que limita el crecimiento de los tallos (10-12 ft o 3-3.7 m) y rendimientos de cono (Agehara 2018). La iluminación artificial se puede utilizar para crear condiciones de día largo ya sea mediante la extensión de horas de luz o la interrupción nocturna. En nuestro campo de lúpulo, hemos demostrado que la extensión de horas de luz con bombillas LED es muy eficaz para inhibir la floración prematura.

\section{Construcción del Sistema de Tutorado para Lúpulo Paso 1. Selección del lugar}

La selección del lugar es una de las decisiones más importantes que determina el éxito o el fracaso del manejo del cultivo de lúpulo, considerando los altos costos de establecimiento y la longevidad de las plantas de lúpulo. En un campo de lúpulo comercial bien manejado, las plantas pueden ser productivas hasta por 20 años (Michigan State University 2015). El diseño ideal tiene hileras orientadas de norte a sur con pendiente hacia el sur. Este diseño permite la captura máxima de luz solar y su distribución uniforme a medida que el sol se mueve de este a oeste. La pendiente orientada al sur maximiza la exposición a la luz solar, lo que calienta el suelo más rápido y mejora el drenaje.

Se recomienda realizar pruebas de suelo para determinar tipo de suelo, pH y las necesidades básicas de nutrientes. El lúpulo crece en una amplia variedad de suelos, pero los ideales son suelos franco-arenosos, profundos y bien drenados (Gent et al. 2019). El buen drenaje es fundamental para el lúpulo, especialmente en Florida, donde las fuertes lluvias son frecuentes durante el verano. El pH óptimo del suelo para la producción de lúpulo es de 6 a 7 (Gent et al. 2019). En nuestro campo de lúpulo, el hierro es la deficiencia de micronutrientes más común, especialmente durante la etapa de crecimiento inicial. Algunos cultivares de lúpulo son altamente susceptibles a los nemátodos agalladores, los cuales son comunes en los suelos de Florida (Desaeger 2018). Por lo tanto, también se recomienda análisis para nemátodos con el objetivo de evitar un sitio con altas poblaciones de ellos.

Los vientos fuertes pueden dañar los tallos de lúpulo retorciéndolos o por fricción con arena. En nuestro campo de lúpulo, hemos observado daños mecánicos en tallos, hojas y conos después de fuertes vientos y lluvia. También se encontró infección causada por varias especies de Fusarium, que típicamente se asoció con daño mecánico en las ramas laterales. Recomendamos instalar cortavientos para minimizar el daño eólico y el riesgo de infección. Otros factores importantes para la selección del lugar incluyen planes de mercadeo, disponibilidad de agua de riego de 
alta calidad, electricidad para iluminación suplementaria y mano de obra.

\section{Paso 2. Preparación del terreno}

Antes de la preparación de las camas de cultivo, el pH del suelo debe ajustarse entre 6 y 7 . Consulte https://edis.ifas. ufl.edu/ss651 para bajar el $\mathrm{pH}$ del suelo (Mylavarapu et al. 2019) o https://edis.ifas.ufl.edu/vh024 para aumentar el pH del suelo (Stephens and Liu 2019). Para conocer las recomendaciones básicas sobre la interpretación de los resultados de análisis de suelo y los cálculos de fertilizantes, consulte https://edis.ifas.ufl.edu/hs1206 (DeValerio et al. 2018).

El distanciamiento típico entre hileras en un campo de lúpulo varía de 12 a $15 \mathrm{ft}$ (3.7-4.6 m). Elija el espacio entre hileras según el tamaño del equipo agrícola que se utilizará en su campo de lúpulos. Si su suelo es bajo en contenido de materia orgánica, recomendamos incorporar compost. El suelo en GCREC es arena fina Myakka, que típicamente tiene contenidos de arena y materia orgánica de $>95 \%$ y $<1.5 \%$, respectivamente. Nosotros agregamos cerca de 2 " (aproximadamente 80 ton/acre o 198 ton/ha) de compost derivado principalmente de desechos de jardín (vertedero del sudeste del condado de Hillsborough, Lithia, FL) a la mitad de nuestro campo de lúpulo. Las plantas crecieron mucho mejor con compost en comparación con aquellas que fueron fertilizadas sólo vía fertirriego. Debido a que los lúpulos desarrollan extensos sistemas radiculares con altas demandas de agua (Nakawuka et al. 2017; Evans 2003; George 2001), recomendamos hacer camas de cultivo bajas e instalar dos líneas de mangueras de goteo por hilera (Figura 2A). En nuestro campo de lúpulo, instalamos mangueras de goteo de material grueso $(0.26 \mathrm{gph}$, distanciamiento de goteros 12 " o $30 \mathrm{~cm}$, diámetro interno de $0.62 "$ o $1.57 \mathrm{~cm}$, grosor de pared de $0.045^{\prime \prime}$ o $0.11 \mathrm{~cm}$ ) que normalmente puede durar más de 20 años. Es importante hacer una pendiente suave hacia el medio de cada pasillo (entre camas) para un buen drenaje. Para el control de malezas, recomendamos usar cobertores de suelo y establecer pastos entre las hileras (Figura 2B). Aplicar un fumigante antes de plantar es una medida preventiva buena para garantizar el establecimiento después de trasplante. (Desaeger 2018).

\section{Paso 3. Construcción del sistema de tutorado}

La construcción de un sistema de tutorado duradero es esencial para la producción de lúpulo. En Florida, el diseño del tutorado y los materiales utilizados deben soportar no sólo el peso de las plantas de lúpulo, sino también los vientos y lluvias fuertes. La Figura 2 muestra los materiales utilizados para construir el sistema de tutorado del campo de lúpulo.

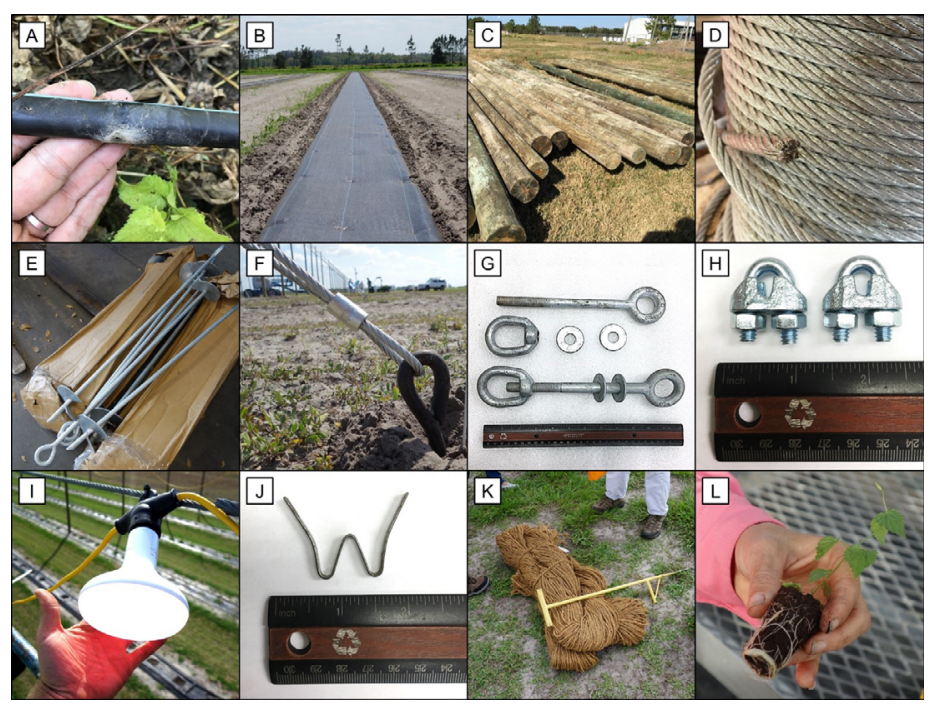

Figura 2. Materiales utilizados para el establecimiento de un campo de lúpulo: A) mangueras de goteo, B) cobertores de suelo, C) postes tratados a presión, D) cables galvanizados de 5/16" (tipo de filamento $7 \times 19)$, E) anclajes, F) cable de anclaje asegurado con un casquillo de aluminio, G) armellas y tuercas, $\mathrm{H}$ ) nudos para cables de metal de $3 / 16^{\prime \prime}$, I) bombilla LED instalada en un cable de $5 / 16^{\prime \prime}, \mathrm{J}$ ) clip en forma de W para la instalación de cuerdas de tutorado, K) cuerdas de fibra de coco y un aplicador de clip en forma de W para la instalación de cuerdas de tutorado, y L) plántula de lúpulo propagada por cultivo de tejidos.

Créditos: Shinsuke Agehara y Aleyda Acosta-Rangel, UF/IFAS

La Figura 3 ilustra los dos diseños de tutorado instalados en el campo de lúpulo de GCREC: tutorado recto y tutorado en "V". El tutorado recto tiene solo un cable por fila de postes, que es para instalar tanto bombillas LED como cuerdas. Por el contrario, el tutorado en " $\mathrm{V}$ " tiene tres cables por fila de postes (Figura 3A): el cable del medio se usa para colgar bombillas LED y los otros dos se utilizan para instalar cuerdas. El tutorado en " $V$ " también tiene cables perpendiculares al central, donde se sostendrán los cables para instalar las cuerdas. Aunque el tutorado en V requiere costos de material más altos que el tutorado recto (consulte la sección "Costos de material” a continuación), su diseño en " $V$ " aprovecha al máximo el espacio de cultivo (Figura 3B). La evaluación del crecimiento y rendimiento de las plantas de lúpulo con los dos diseños está en proceso.

Se recomiendan los postes tratados a presión porque son más resistentes a la descomposición natural y duran más que la madera sin tratar. Nuestros postes perimetrales tienen $6 "(15.2 \mathrm{~cm})$ de diámetro y los postes interiores tienen 5 " $(12.7 \mathrm{~cm})$ de diámetro. Todos nuestros postes miden $25 \mathrm{ft}(7.62 \mathrm{~m})$ de largo y están enterrados $5 \mathrm{ft}(1.5 \mathrm{~m})$ en el suelo. En los campos comerciales de lúpulo, es común 
inclinar los postes perimetrales para aumentar la fuerza de sujeción (Arnett 2018). Aunque todos nuestros postes fueron instalados perpendiculares al suelo para facilitar la futura expansión del campo (Figuras 1 y 4C), no se han observado daños estructurales.

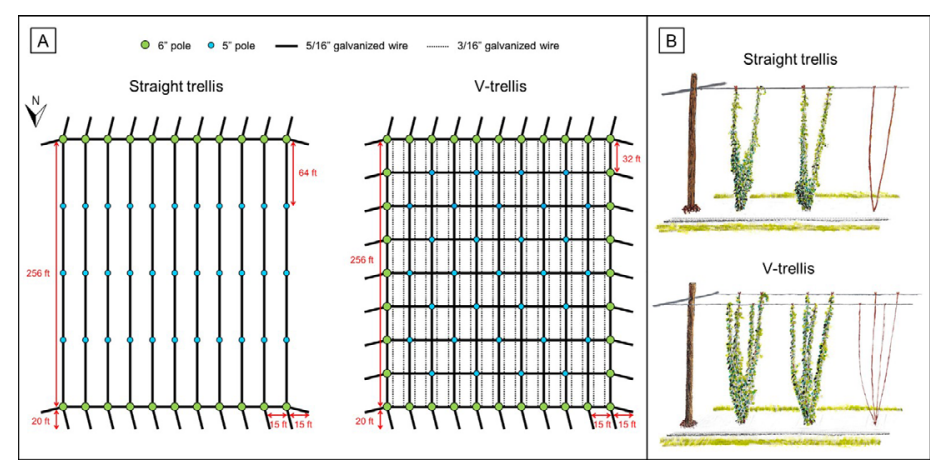

Figura 3. Diseños de tutorado recto y en "V" usados para el campo de lúpulo de GCREC: A) planos del tutorado y B) diagramas del tutorado. Créditos: Shinsuke Agehara y Aleyda Acosta-Rangel, UF/IFAS

Los cables de tutorado que se utilizan comúnmente en los campos comerciales de lúpulos son cables de acero galvanizado con hebras de $1 \times 7$ (una hebra con 7 hilos) o $7 \times 19$ ( 7 hebras con 19 hilos en cada hebra) (Arnett 2018). La resistencia de un cable galvanizado depende de su grado, diámetro y tipo de hebra. Los cables galvanizados que se usan en nuestro campo de lúpulo son de $5 / 16$ " o 3/16" de diámetro con un tipo de filamento de $7 \times 19$ (Figura 2D). Usamos cables con diferentes diámetros según el diseño del tutorado (Figura 3A). Los cables de tutorado se pasaron a través de orificios pretaladrados en los postes. Los orificios se hicieron con una broca de $5 / 8$ " a $2 \mathrm{ft}(61 \mathrm{~cm})$ de la parte superior del poste (Figura 4B). También se colocaron cables desde los postes perimetrales hacia los anclajes.

\section{Paso 4. Instalación de iluminación suplementaria}

El uso de iluminación suplementaria que asegura el buen crecimiento vegetativo antes de la floración es fundamental para una producción exitosa de lúpulo en Florida. En el campo de lúpulo de GCREC, instalamos bombillas LED (GreenPower LED flowering DR/ W, Philips) en los cables centrales del tutorado (Figura 5A). Nuestras recomendaciones actuales son espaciar las bombillas LED a $20 \mathrm{ft}(6.1 \mathrm{~m})$ de distancia en un patrón intercalado (138 bombillas por acre o 341 bombillas por hectárea) y operarlas durante 6 horas por noche ( $>17$ horas de luz por día) durante 7 a 9 semanas o hasta que las plantas desarrollen suficiente crecimiento vegetativo. Las plantas normalmente comienzan a desarrollar botones florales 5 días después de apagar la iluminación suplementaria.

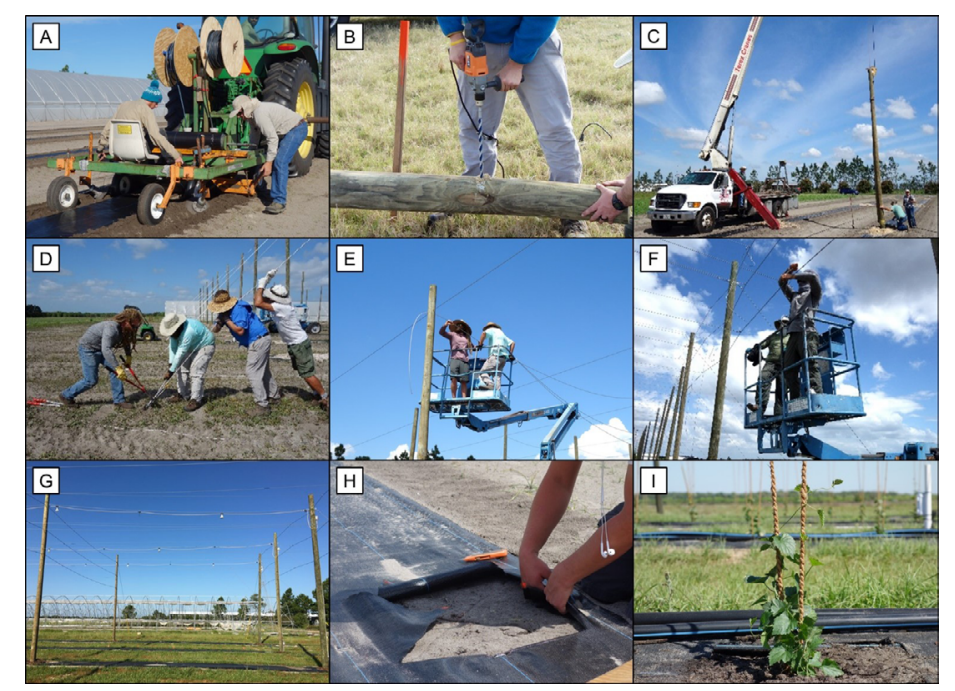

Figura 4. El proceso de establecimiento del campo de lúpulo en el UF/IFAS GCREC: A) instalación del cobertor de suelo y manguera de goteo, B) perforación de un agujero en un poste de 6", C) instalación del poste con una grúa, D) instalación de un cable de anclaje, E) instalación de cables de $5 / 16$ ", F) instalación de cables de $3 / 16^{\prime \prime}$ para un tutorado en " $V$ ", G) bombillas LED instaladas en cables de 5/16", H) corte en el cobertor de suelo para el hoyo de plantación, y l) una plántula de lúpulo recién trasplantada.

Créditos: Shinsuke Agehara y Aleyda Acosta-Rangel, UF/IFAS

Actualmente estamos evaluando el número óptimo de bombillas LED necesarias para controlar la floración. Los distanciamos a 9 y $20 \mathrm{ft}(2.7$ y $6.1 \mathrm{~m})$ en 2018 y 2019 , respectivamente, y se demostró que el distanciamiento de $20 \mathrm{ft}$ es tan efectivo como el de $9 \mathrm{ft}$.

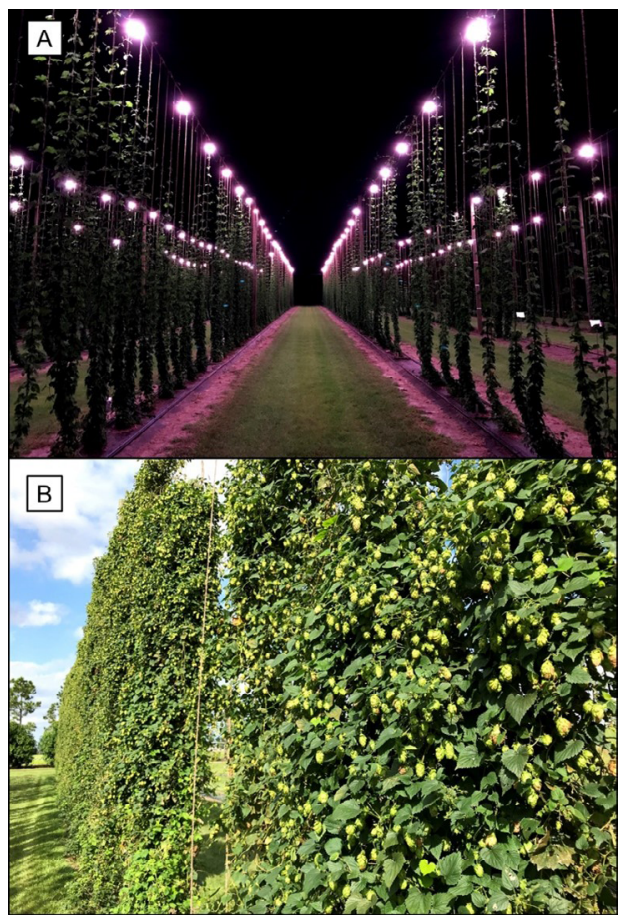

Figura 5. Plantas de lúpulo cultivadas con iluminación suplementaria en el campo de lúpulo de GCREC: A) plantas de lúpulo iluminadas con bombillas LED por la noche y B) plantas de lúpulo que alcanzaron la parte superior del cable central y producen muchos conos aproximadamente un mes después de apagar las bombillas LED. Créditos: Shinsuke Agehara, UF/IFAS 


\section{Paso 5. Trasplante}

Establecer un campo de lúpulo con material vegetal limpio es esencial para una producción exitosa de lúpulo. Florida no tiene un registro histórico de patógenos típicos del lúpulo como el mildiú velloso del lúpulo (Pseudoperonospora humuli), el mildiú polvoso del lúpulo (Podosphaera macularis) o el virus latente del lúpulo, todos ellos son específicos para este hospedero (Vallad et al. 2019). Debido a que el clima de Florida es propicio para la propagación de enfermedades, es fundamental evitar la posibilidad de introducir estos patógenos en el estado. Por esta razón, se recomienda encarecidamente a los productores de Florida que utilicen plantas propagadas por cultivo de tejidos y que estén certificadas libres de virus. No recomendamos el uso de rizomas, ya que pueden estar infectados con patógenos fúngicos o virales. Nuestro campo de lúpulo se estableció utilizando plantas propagadas por cultivo de tejidos de un vivero local (Agri-Starts, Apopka, FL). Hicimos hoyos de plantación de $12 " \times 12 "(30 \times 30 \mathrm{~cm})$ cortando el cobertor de suelo y plantamos dos plántulas por hoyo. Las cuerdas de tutorado deben instalarse antes o inmediatamente después de la siembra. Según nuestra evaluación de variedades de lúpulos, 'Cascade' es la variedad más prometedor en términos de rendimiento, calidad de cono y tolerancia a nemátodos (Desaeger 2018).

\section{Costos de materiales}

Los costos de establecimiento del campo de lúpulo dependen del diseño del tutorado, los materiales utilizados y los recursos que puedan tener los productores, como el equipo y la infraestructura agrícolas. La Tabla 1 muestra los costos por acre de los materiales utilizados para construir nuestro campo de lúpulo con dos diseños de tutorado diferentes. El costo total del material para el establecimiento de nuestro campo de lúpulo fue de $\$ 15,780$ para el sistema de tutorado recto y $\$ 18,687$ para el tutorado en "V". Tenga en cuenta que estos costos no incluyen tarifas de envío, que pueden costar hasta $\$ 1,000$ por acre, ni costos laborales. La principal diferencia de costo radica en que el tutorado en "V" requiere aproximadamente un $20 \%$ más de postes y un $370 \%$ más de cables que el tutorado recto. La mayoría de los productores comerciales de lúpulo en Washington usan tutorado en "V" de $18 \mathrm{ft}$ (5.5 metros) de altura (Dodds 2017). El costo subtotal de los materiales de construcción del tutorado para nuestro diseño en "V" fue de $\$ 8,225$ por acre, que fue $\$ 1,230$ más alto que el de un tutorado similar en la región noroeste del Pacífico $(\$ 6,995)$ (Washington State University 2016). Las principales diferencias de costos son debido a los postes y cables. El costo total del material para el establecimiento de nuestro campo de lúpulo fue relativamente alto debido a que algunos artículos específicos solo se requieren en Florida. Entre esos elementos, la instalación de iluminación suplementaria es el gasto más grande, con un total de $\$ 5,398$ por acre. El costo puede aumentar significativamente dependiendo de la disponibilidad de materiales para las conexiones eléctricas en la propiedad (por ejemplo, transformadores).

\section{Literatura Citada}

Agehara, S. 2018. “Tricking Hop Plants Using LED Lights." Berry Vegetable Times 18:4-5.

Arnett, M. 2018. "Choosing between a Wooden Pole or a Galvanized Steel Post Design-A Decision to Make When Designing a Hop Yard Trellis System for Growing Plants in a V-Bine Configuration." Accessed November 19, 2019. https://soncotube.com/wp-content/uploads/2019/01/ Designing-and-Installing-a-Hop-Yard-Trellis-System-1-1. pdf

Brewers Association. 2018. "Florida's Craft Beer Sales and Production Statistics, 2018." Accessed November 19, 2019. https://www.brewersassociation.org/statistics-and-data/ state-craft-beer-stats/?state $=\mathrm{FL}$

Desaeger, J. 2018. "Nematodes Parasitizing Hops in Florida.” EDIS 2018 (6). https://doi.org/10.32473/ edis-in1229-2018

DeValerio, J., D. Nistler, R. Hochmuth, and E. Simonne. 2018. "Fertigation for Vegetables: A Practical Guide for Small Fields.” EDIS 2012 (10). https://edis.ifas.ufl.edu/ hs 1206

Dodds, K. 2017. “Hops: A Guide for New Growers." NSW Department of Primary Industries. Accessed November 19, 2019. https://www.dpi.nsw.gov.au/_data/assets/ pdf_file/0007/712717/hops-guide-for-new-growers.pdf

Evans, R. 2003. Hop Management in Water-Short Periods. EM4816. Pullman, WA: Washington State University Cooperative Extension. Accessed November 14, 2019. http://www.willingham-nurseries.co.uk/books/Hops/ em4816.pdf

Gent, D., R. Sirrine, and H. Darby. 2019. "Nutrient Management and Imbalances." Hop Growers of America. Accessed November 19, 2019. https://www.usahops.org/cabinet/ data/9.pdf 
George, A. E. 2001. "Crop Profiles for Hops in Washington." Accessed November 14, 2019. http://content.libraries.wsu. edu/index.php/utils/getfile/collection/cahnrs-arch/id/414/ filename/76456182432004_Hops3PM.pdf

Hop Growers of America. 2019. "2018 Statistical Report.” Hop Growers of America. Accessed November 19, 2019. https://www.usahops.org/img/blog_pdf/168.pdf

International Hop Growers' Convention (IHGC). 2018. "Economic Commission - Summary Reports." Accessed November 19, 2019. http://www.hmelj-giz.si/ihgc/ doc/2018\%20MAY\%20IHGC\%20EC\%20Reports.pdf

Iskra, A. E., S. R. Lafontaine, K. M. Trippe, S. T. Massie, C. L. Phillip, M. C. Twomey, T. H. Shellhammer, and D. H. Gent. 2019. "Influence of Nitrogen Fertility Practices on Hop Cone Quality.” J. Am. Soc. Brew. Chem. 77:199-209.

Mylavarapu, R., G. Hochmuth, C. Mackowiak, A. Wright, and M. Silveira. 2019. "Lowering Soil $\mathrm{pH}$ to Optimize Nutrient Management and Crop Production." EDIS 2016 (1). https://edis.ifas.ufl.edu/ss651

Nakawuka, P., T. Peters, S. Kenny, and D. Walsh. 2017. "Effect of Deficit Irrigation on Yield Quantity and Quality, Water Productivity and Economic Returns of Four Cultivars of Hops in the Yakima Valley, Washington State." Industrial Crops and Products 98:82-92.

Siegle, L. 2017. "Growing Hops in the South Atlantic: The Basics." Accessed January 6, 2020. https://ext.vt.edu/ content/dam/ext_vt_edu/topics/agriculture/commercialhorticulture/hops/beginnersSAHC2017revised.pdf

Sirrine, R., E. Lizotte, D. Brown, T. O'Brien, and A. Leach. 2014. Estimated Cost of Producing Hops in Michigan. E3236. East Lansing, MI: Michigan State University Extension. Accessed November 19, 2019. https://www.canr.msu. edu/uploads/234/71501/MI_Hops_cost_of_production_Bulletin-E3236.pdf

Stephens, J., and G. Liu. 2019. "Soil Preparation and Liming for Vegetable Gardens.” EDIS 2013 (6). https://edis.ifas.ufl. edu/vh024

Thomas, G. G., and W. W. Schwabe. 1969. "Factors Controlling Flowering in the Hop (Humulus lupulus L.)." Annals of Botany 33:781-793.
US Department of Agriculture-Agricultural Research Services. 2007. "Hop Cultivar Descriptors." Accessed November 19, 2019. https://www.ars.usda.gov/pacific-westarea/corvallis-or/forage-seed-and-cereal-research/people/ john-henning/cultindex/

US Department of Agriculture-National Agricultural Statistics Service. 2019a. "Hop Stocks." Accessed November 19, 2019. https://downloads.usda.library.cornell.edu/usdaesmis/files/sb397825r/73666h42j/5t34sz16b/hops0919.pdf

US Department of Agriculture-National Agricultural Statistics Service. 2019b. "Press Release." Accessed November 19, 2019. https://www.usahops.org/img/blog_pdf/237.pdf

Vallad, G., Y. Desaeger, H. Smith, Z. Deng, and S. Agehara. "Florida Hop Trials-Keeping Ahead of Pests and Diseases." VSCNews. Accessed November 14, 2019. http://vscnews.com/ florida-hop-trials-keeping-ahead-of-pests-and-diseases/

Washington State University. 2016. 2015 Estimated Cost of Establishing and Producing Hops in the Pacific Northwest. TB38. Pullman, WA: Washington State University Extension. Accessed November 19, 2019. http://ses.wsu.edu/ wp-content/uploads/2018/10/TB38.pdf

Yakima Chief Hops. 2019. "Yakima Chief Hops Varieties." Accessed November 19, 2019. https://shop.yakimachief. com/media/wysiwyg/Yakima_Chief_Hops_Varieties.pdf 
Tabla 1. Costos de Materiales para Establecer un Campo de Lúpulos de un Acre Usando Tutorado Recto y Tutorado en "V" en Florida.'

\begin{tabular}{|c|c|c|c|c|c|}
\hline \multirow[t]{2}{*}{ Material } & \multirow{2}{*}{$\begin{array}{l}\text { Precio } \\
\text { unitario }\end{array}$} & \multicolumn{2}{|c|}{ Cantidad } & \multicolumn{2}{|c|}{ Costo total } \\
\hline & & Tutorado recto & Tutorado en "V" & Tutorado recto & Tutorado en "V" \\
\hline \multicolumn{6}{|l|}{ Preparación del terreno } \\
\hline $\begin{array}{l}\text { Manguera de riego }(1000 \mathrm{ft} / \\
\text { rollo) }\end{array}$ & $\$ 150.00$ & 6 & 6 & $\$ 900$ & $\$ 900$ \\
\hline Corteza de pino ( $\left.2 \mathrm{ft}^{3} / \mathrm{bolsa}\right)$ & $\$ 2.50$ & 44 & 44 & $\$ 110$ & $\$ 110$ \\
\hline Cobertor de suelo (300 ft/rollo) & $\$ 69.19$ & 11 & 11 & $\$ 761$ & $\$ 761$ \\
\hline Piezas misceláneas para riego & & & & $\$ 500$ & $\$ 500$ \\
\hline Subtotal & & & & $\$ 2,271$ & $\$ 2,271$ \\
\hline \multicolumn{6}{|l|}{ Construcción del tutorado } \\
\hline Poste tratado a presión de $6 "$ & $\$ 76.50$ & 22 & 36 & $\$ 1,683$ & $\$ 2,754$ \\
\hline Poste tratado a presión de $5 "$ & $\$ 58.50$ & 33 & 31 & $\$ 1,931$ & $\$ 1,814$ \\
\hline $\begin{array}{l}\text { Cable galvanizado de } 5 / 16^{\prime \prime} \\
(5,000 \mathrm{ft} / \mathrm{rollo})^{2}\end{array}$ & $\$ 0.36$ & 3,900 & 5,300 & $\$ 1,404$ & $\$ 1,908$ \\
\hline $\begin{array}{l}\text { Cable galvanizado de } 3 / 16 \\
(5,000 \mathrm{ft} / \mathrm{rollo})^{2}\end{array}$ & $\$ 0.17$ & 0 & 5,300 & $\$ 0$ & $\$ 901$ \\
\hline Armella de $5 / 8^{\prime \prime}$ & $\$ 5.24$ & 26 & 40 & $\$ 136$ & $\$ 210$ \\
\hline Tuerca de $5 / 8^{\prime \prime}$ & $\$ 3.47$ & 26 & 40 & $\$ 90$ & $\$ 139$ \\
\hline Casquillo de aluminio de $5 / 16^{\prime \prime}$ & $\$ 0.40$ & 78 & 120 & $\$ 31$ & $\$ 48$ \\
\hline Nudo para cables de metal 3/16" & $\$ 1.10$ & 0 & 44 & $\$ 0$ & $\$ 48$ \\
\hline Anclaje 5/8" & $\$ 7.59$ & 26 & 40 & $\$ 197$ & $\$ 304$ \\
\hline Otros (misceláneos) & & & & $\$ 100$ & $\$ 100$ \\
\hline Subtotal & & & & $\$ 5,573$ & $\$ 8,225$ \\
\hline \multicolumn{6}{|l|}{ Iluminación suplementaria } \\
\hline Bombillas LED & $\$ 23.25$ & 138 & 138 & $\$ 3,209$ & $\$ 3,209$ \\
\hline Cables de electricidad (100 ft) & $\$ 66.36$ & 33 & 33 & $\$ 2,190$ & $\$ 2,190$ \\
\hline Subtotal & & & & $\$ 5,398$ & $\$ 5,398$ \\
\hline \multicolumn{6}{|l|}{ Trasplante } \\
\hline $\begin{array}{l}\text { Cuerdas de fibra de coco ( } 75 \mathrm{lb} \text {, } \\
3,200 \text { cuerdas/fardo) }\end{array}$ & $\$ 0.12$ & 1,848 & 3,696 & $\$ 222$ & $\$ 444$ \\
\hline $\begin{array}{l}\text { Clips en forma de W (1000 } \\
\text { unidades/caja) }\end{array}$ & $\$ 33.00$ & 1 & 2 & $\$ 33$ & $\$ 66$ \\
\hline Aplicador de clips en forma de W & $\$ 65.00$ & 1 & 1 & $\$ 65$ & $\$ 65$ \\
\hline $\begin{array}{l}\text { Plántulas de lúpulos propagadas } \\
\text { por cultivo de tejidos }{ }^{4}\end{array}$ & $\$ 1.20$ & 1,848 & 1,848 & $\$ 2,218$ & $\$ 2,218$ \\
\hline Subtotal & & & & $\$ 2,538$ & $\$ 2,793$ \\
\hline TOTAL & & & & $\$ 15,780$ & $\$ 18,687$ \\
\hline \multicolumn{6}{|c|}{$\begin{array}{l}{ }^{1} \text { Los planos de tutorado del campo de lúpulos están ilustrados en la Figura } 3 \text {. } \\
{ }^{2} \text { El precio unitario es por pie. } \\
{ }^{3} \text { El precio unitario es por cuerda. } \\
{ }^{4} \text { El número de plántulas por acre es } 1848 \text { ( } 924 \text { hoyos de plantación/acre } \times 2 \text { plántulas/siembra). Los hoyos de plantación están distanciados a } \\
3 \mathrm{ft} \text { dentro de cada hilera ( } 84 \text { hoyos de plantación en una hilera de } 256 \mathrm{ft} \text {. }\end{array}$} \\
\hline
\end{tabular}

